\title{
Commentary: Correlation between Patent Foramen Ovale, Cerebral "Lesions" and Neuropsychometric Testing in Experienced Sports Divers: Does Diving Damage the Brain?
}

\author{
Emmanuel Gempp * \\ Diving and Hyperbaric Medicine, French Armed Forces Health Service, Toulon, France
}

Keywords: diving, long-term effects, persistent foramen ovale, brain MRI, cerebral embolism

\section{OPEN ACCESS}

Edited by:

Guy Cheron,

Université libre de Bruxelles, Belgium

Reviewed by:

François Guerrero,

University of Western Brittany, France

Patrice Jissendi Tchofo,

Saint-Pierre University Hospital,

Belgium

Jacek Kot,

Gdañsk Medical University, Poland

*Correspondence:

Emmanuel Gempp gempp@netc.fr

Specialty section: This article was submitted to Movement Science and Sport

Psychology,

a section of the journal

Frontiers in Psychology

Received: 05 June 2016 Accepted: 08 August 2016 Published: 24 August 2016

Citation:

Gempp E (2016) Commentary: Correlation between Patent Foramen

Ovale, Cerebral "Lesions" and

Neuropsychometric Testing in Experienced Sports Divers: Does

Diving Damage the Brain?

Front. Psychol. 7:1254

doi: 10.3389/fpsyg.2016.01254

\section{A commentary on}

Correlation between Patent Foramen Ovale, Cerebral "Lesions" and Neuropsychometric Testing in Experienced Sports Divers: Does Diving Damage the Brain?

by Balestra, C., and Germonpré, P. (2016). Front. Psychol. 7:696. doi: 10.3389/fpsyg.2016.00696

After the careful reading of a recent and interesting article published by Balestra and Germonpré (2016) suggesting the lack of correlation between white-matter brain MRI hyperintensities, neuro-psychometric alterations, and the presence of persistent foramen ovale (PFO) screened by contrast transoesophageal echocardiography in recreational scuba divers, I would like to provide additional precisions and also discuss some of their findings.

The paper that referred to "Gempp et al. (2008)" is inadequate. The reader should rather consider the following reference: Gempp et al. (2010). In that study, we clearly showed that the number and size of cerebral MRI signal abnormalities in 34 military divers who strictly adhere to decompression procedures were closely related to the grade of right-to-left shunt determined by transcranial Doppler. Our results were also in line with the conclusions of 2 other radiological investigations, not cited by Balestra and Germonpré (2016) (Schwerzmann et al., 2001; Billinger et al., 2011), suggesting a potential causal relationship between focal cerebral hyperintensities and the presence of this anatomical predisposition (i.e., PFO). In their work, Balestra and Germonpré (2016) reported only 5 out of 42 divers having a total of 5 lesion-like hyperintense signals detected by brain MRI while nearly $64 \%$ of them (including 3 out of 5 divers with positive imaging) exhibited a PFO of varying importance. Neither the presence of PFO nor the existence of signal abnormalities were associated with an impairment of neuro-psychometric tests battery in this population. Although this outcome may be seen as a reassuring message for the scientific community, it should be interpreted with caution since this negative result is based on a very small number of MRI findings, hence making difficult to find a reliable correlation between the extent of radiological brain changes, the performance of the neuro-psychometric tests used, and the patency of PFO. In parallel, the authors did not mention, probably for publication deadline reasons, the results of a recent metaanalysis comprising 7 medium/high quality studies that revealed a highly significant increase in prevalence of cerebral hyperintense spots found with MRI in 98 out of 279 healthy divers (mainly professional) compared to 44 out of 232 non-diver controls (OR 2.65, 95\% CI 1.71-4.39, $p<0.001$; Connolly and Lee, 2015). 
Anyway, the authors should be strongly encouraged and acknowledged for their excellent work reinforcing the notion that uneventful repeated diving exposure may have a deleterious impact on higher cognitive functions of scuba divers. Whether the hemodynamically relevance of PFO favors the development of long-term brain damage rather than diving alone remains, however, to be elucidated. A longitudinal follow-up study of brain MRI hyperintensities prevalence and neuro-psychometric

\section{REFERENCES}

Balestra, C., and Germonpré, P. (2016). Correlation between patent foramen ovale, cerebral "lesions" and neuropsychometric testing in experienced sports divers: does diving damage the brain? Front. Psychol. 7:696. doi: 10.3389/fpsyg.2016.00696

Billinger, M., Zbinden, R., Mordasini, R., Windecker, S., Schwerzmann, M., Meier, B., et al. (2011). Patent foramen ovale closure in recreational divers: effect on decompression illness and ischaemic brain lesions during long term follow-up. Heart 97, 1932-1937. doi: 10.1136/heartjnl-2011-300436

Connolly, D. M., and Lee, V. M. (2015). Odds ratio meta-analysis and increased prevalence of white-matter injury in scuba divers. Aerosp. Med. Hum. Perform. 86, 928-935. doi: 10.3357/AMHP.4392.2015

Gempp, E., Blatteau, J. E., Stephant, E., Pontier, J. M., Constantin, P., and Pény, C. (2008). MRI findings and clinical outcome in 45 divers with spinal cord decompression sickness. Aviat. Space Environ. Med. 79, 1112-1116. doi: 10.3357/ASEM.2376.2008

Gempp, E., Sbardella, F., Stephant, E., Constantin, P., De Maistre, S., Louge, P., et al. (2010). Brain MRI signal abnormalities and right-to-left shunting in alterations in relation with the presence of PFO in a carefully selected group of scuba divers could be the best way to make progress in this area.

\section{AUTHOR CONTRIBUTIONS}

The author confirms being the sole contributor of this work and approved it for publication.

asymptomatic military divers. Aviat. Space Environ. Med. 81, 1008-1012. doi: 10.3357/ASEM.2786.2010

Schwerzmann, M., Seiler, C., Lipp, E., Guzman, R., Lövblad, K. O., Kraus, M., et al. (2001). Relation between directly detected patent foramen ovale and ischemic brain lesions in sport divers. Ann. Intern. Med. 134, 21-24. doi: 10.7326/0003-4819-134-1-200101020 00009

Conflict of Interest Statement: The author declares that the research was conducted in the absence of any commercial or financial relationships that could be construed as a potential conflict of interest.

Copyright (C) 2016 Gempp. This is an open-access article distributed under the terms of the Creative Commons Attribution License (CC BY). The use, distribution or reproduction in other forums is permitted, provided the original author(s) or licensor are credited and that the original publication in this journal is cited, in accordance with accepted academic practice. No use, distribution or reproduction is permitted which does not comply with these terms. 